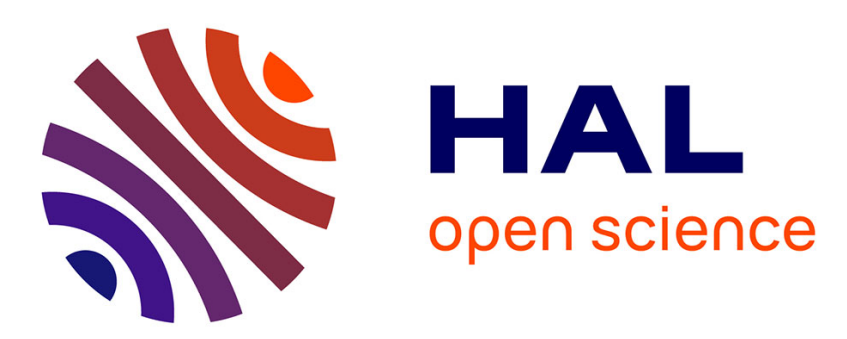

\title{
Le NIMBY 1 est mort. Vive la résistance éclairée Le cas de l'opposition à un projet de décharge, Essonne, France.
}

Léa Sébastien

\section{To cite this version:}

Léa Sébastien. Le NIMBY 1 est mort. Vive la résistance éclairée Le cas de l'opposition à un projet de décharge, Essonne, France.. Sociologies pratiques, 2013, 27 (2), pp.145-165. hal-01511034

\section{HAL Id: hal-01511034 \\ https://hal-univ-tlse2.archives-ouvertes.fr/hal-01511034}

Submitted on 20 Apr 2017

HAL is a multi-disciplinary open access archive for the deposit and dissemination of scientific research documents, whether they are published or not. The documents may come from teaching and research institutions in France or abroad, or from public or private research centers.
L'archive ouverte pluridisciplinaire HAL, est destinée au dépôt et à la diffusion de documents scientifiques de niveau recherche, publiés ou non, émanant des établissements d'enseignement et de recherche français ou étrangers, des laboratoires publics ou privés. 


\title{
Le NIMBY $^{1}$ est mort. Vive la résistance éclairée
}

\section{Le cas de l'opposition à un projet de décharge, Essonne, France.}

Article paru dans Sociologies Pratiques vol 27, $n^{\circ} 2$. Pour citer cet article :

Sébastien, Léa. "Le nimby est mort. Vive la résistance éclairée: le cas de l'opposition à un projet de décharge, Essonne, France." Sociologies pratiques 27, no. 2 (2013): 145-165.

\section{Résumé}

Le NIMBY est présenté comme un front de refus systématique aux projets industriels générateurs de nuisances, fondé sur l'ignorance et l'égoïsme. Dans certains cas, il n'en est rien. Voici l'exemple d'une résistance organisée face à un projet de décharge sur la petite commune rurale de St-Escobille (Essonne, France). En décortiquant le processus de contestation, cet article montre que le conflit environnemental a conduit au renforcement de quatre types de capitaux : le capital social, le capital savant, le capital patrimonial et le capital politique. Ce type d'opposition locale, que nous appelons « résistance éclairée » peut alors être l'expression d'un mouvement social enrichissant la démocratie et créant de nouvelles valeurs territoriales. La première partie de cet article explicite le phénomène NIMBY ainsi que ses différentes dimensions. On se propose ensuite d'analyser de quelle manière la résistance à la décharge s'organise et comment elle amène à une reconfiguration du paysage social, en explicitant les quatre types de capitaux. La dernière partie remet en cause le terme de NIMBY pour aborder les incertitudes autour des problématiques environnementales et analyse les nouvelles formes de légitimités pour définir l'intérêt général.

\section{Mots-clés}

CSDU, NIMBY, risque, conflit environnemental, controverse, mobilisation, déchet, résistance, capital politique, Beauce.

\section{Death of NIMBY. Awakening of argumented resistance.}

\section{The case of opposition to a landfill project, Essonne, France.}

\begin{abstract}
NIMBY supporters are often presented as ignorant and selfish citizens, systematically against any form of industry. Here is an example of an organized resistance to a project of landfill in the village of St-Escobille (Essonne, France). Because it does not fall into the NIMBY framework, this particular case brings interesting matters on the question. Through an analysis of the construction of the opposition movement, this paper shows that environmental conflicts reinforce four types of capitals on the territory: social capital, intellectual capital, patrimonial capital, political capital. We call this type of local opposition "argumented resistance", which can be the extension of social movements enriching democracy and building new territorial values. After reviewing the various dimensions of the NIMBY phenomena, this paper describes organizational aspects of the local resistance, together with unsuspected reconfiguration of the social relations in the community. Our discussion puts into question the notion of NIMBY regarding environmental uncertainties and analyses the rising forms of legitimacy to define general interest.
\end{abstract}

\section{Key words}

Landfill, NIMBY, risk, environmental conflict, controversy, mobilization, waste, resistance, political capital, Beauce.

\footnotetext{
${ }^{1}$ Not In My Back Yard - traduction de : pas dans mon jardin
} 


\section{Introduction}

Le traitement des déchets figure parmi les priorités en matière de développement durable. Pourtant, environ $60 \%$ des déchets ménagers sont encore mis en décharge, une option située au bas de l'échelle hiérarchique des techniques à utiliser en matière de gestion environnementale des déchets. Depuis le 1er juillet 2002, les $\mathrm{CSDU}^{2}$ ne sont autorisés qu'à recevoir des déchets dits ultimes, « déchets qui ne peuvent plus être traités dans les conditions techniques et économiques du moment ${ }^{3}{ }^{3}$, une définition imprécise qui permet que la mise en décharge soit toujours prépondérante. Leur exploitation génère des nuisances connues, le bruit, l'odeur et des effluents liquides et/ou gazeux : un mélange hétérogène qui évolue chimiquement, biologiquement, mécaniquement, mais le coût immédiat de l'enfouissement est si compétitif que ce processus est fréquemment adopté. Les controverses s'accumulent et nombre de scandales ont d'ailleurs éclaté autour de la gestion des décharges, lesquels ont fragilisé la légitimité des entrepreneurs comme des responsables publics.

Dans ce contexte, l'annonce de la création d'une décharge sur un territoire suscite de vifs mouvements d'opposition de la part des acteurs locaux qui entendent examiner la légitimité du projet, mouvements automatiquement associés au NIMBY, ou parfois NIMEY4 pour les élus, phénomène présenté comme privilégiant les libertés individuelles par rapport au bien commun. Toutefois, I'histoire est-elle toujours aussi simple ? Notre cas d'étude est un projet de CSDU sur la petite commune rurale de St-Escobille, en Essonne. A partir de I'analyse de I'organisation et la construction du mouvement d'opposition, nous testons I'hypothèse selon laquelle certaines contestations locales ne peuvent être réduites au NIMBY et permettent d'enrichir la démocratie en la dotant de valeurs sociales, cognitives, patrimoniales et politiques. Notre première partie s'attachera à expliciter le phénomène NIMBY puis dans un second temps, il s'agira d'analyser précisément le cas de la décharge prévue à St-Escobille et l'organisation des acteurs du territoire dans leur opposition au projet, laquelle dépasse de loin le phénomène de NIMBY. Création de liens sociaux, de connaissances, de valeurs patrimoniales et politiques, nous proposons la notion de « résistance éclairée ». A St-Escobille, devant un projet a priori inapproprié, une masse se soulève, masse où se mêle savoirs profanes et savoirs savants, masse qui s'oppose et qui propose.

\section{Le NIMBY a-t-il toujours un sens?}

Les mouvements d'opposition aux projets générateurs de nuisances sont le plus souvent associés au phénomène NIMBY, bien connu des industriels. Il englobe l'ensemble des pratiques sociales d'opposition à l'implantation ou à l'extension d'installations de nature industrielle pour des raisons de modifications, réelles, prévisibles ou supposées du proche environnement (Maillebouis, 2003). Le NIMBY est caractérisé par une opposition locale intense, parfois émotionnelle et souvent catégorique à une proposition qui entraînera, selon les résidents, des effets négatifs (Wolsink, 2000). Il a lieu lorsque les coûts et les risques d'un projet tels que les impacts sur la santé humaine, sur la qualité de l'environnement ou sur la valeur des propriétés sont concentrés géographiquement tandis que les bénéfices reviennent à une population plus large et plus dispersée.

L'origine du phénomène NIMBY prend place dans les années 70 quand les White Anglo-Saxon Protestants (WASP) militent pour refuser de nouvelles constructions aux abords de leur jardin arrière (backyard garden), lieu d'échanges avec les voisins et de convivialité des banlieues riches américaines. Le NIMBY correspond alors aux mobilisations des catégories privilégiées pour reléguer les infrastructures impactantes ailleurs, c'est-à-dire vers des espaces défavorisés. Et c'est ce qui s'est produit. Les gouvernements et les entreprises ont longtemps construit des projets non désirés et/ou risqués dans des quartiers pauvres sans prendre en compte l'avis de la population. Afin de défendre leur voisinage, leur

\footnotetext{
${ }^{2}$ Centre de Stockage de Déchets Ultimes

${ }^{3}$ Loi du 13 juillet 1992 relative aux installations classées pour la protection de l'environnement

${ }^{4}$ Not In My Election Year - pas l'année de mon élection
} 
santé, leur sécurité et leur mode de vie, les communautés locales ont dû se regrouper pour tenter de contester certaines de ces décisions. Si les premiers mouvements de contestation ne sont pas pris au sérieux, peu à peu les opposants gagnent du terrain et commencent à effrayer élus comme promoteurs.

La littérature scientifique traitant explicitement du phénomène NIMBY (publiée depuis les années 80 ) est majoritairement nord-américaine et en présente deux facettes radicalement différentes, dont la plus classique est hautement critique vis-à-vis des Nimbistes. Véritables grains de sable dans la mise en œuvre de projets censés profiter au plus grand nombre, la multiplication des mouvements NIMBY est souvent présentée comme un obstacle à l'action publique, et plus encore comme un danger pour l'intérêt général. Les Nimbistes apparaissent comme des défenseurs d'intérêts privés, agissant au nom d'une logique individualiste, et sont caractérisés par un défaut de citoyenneté, un déni du politique, entendu comme action ayant pour visée le bien commun (Dziedzcki, 2003). Le terme NIMBY est alors surutilisé par les aménageurs cherchant à affaiblir les opposants à leurs projets, ce qui enferme les mouvements d'opposants dans une position illégitime.

Dans cette veine, Kraft \& Clary, 1991, présentent plusieurs facteurs comme étant à l'origine d'une opposition forte à un projet local : (1) la méfiance envers les porteurs du projet ; (2) l'information limitée sur le problème; (3) une vision locale des risques entraînés par un projet; (4) une évaluation émotionnelle des propositions d'implantation ; (5) une aversion généralisée et particulière du risque. Les détracteurs du phénomène NIMBY expliquent que, suite au développement de ces mouvements sociaux et politiques, des projets essentiels sont devenus impossible à implanter, retardant le développement économique et empêchant la mise en place de solutions techniques supérieures (Dear, 1992). Ainsi peuton lire dans cette littérature scientifique : «Le syndrome NIMBY est une maladie mentale récurrente qui continue à infecter la société »(Piller, 1991) ou « les Nimbistes érodent égoïstement les droits de la communauté »(Brion, 1991). Selon Freudenberg \& Pastor (1992), les réactions NIMBY sont guidées par deux facteurs : I'ignorance (les promoteurs doivent alors éduquer les populations) et par l'égoïsme (la réponse se trouve alors dans la compensation financière des dommages subis). Mais les auteurs soulignent également une autre motivation possible de protestation qu'est la prudence. Dans ce cas, les Nimbistes dénoncent directement les défaillances de I'Etat en matière de décision, de participation et remettent en cause les idées de progrès et de développement. Il s'agit là d'une vision alternative du NIMBY, toujours minoritaire mais néanmoins montante, qui le présente comme une forme légitime de redéfinition de l'intérêt général.

C'est cette dernière attitude que nous rejoignons ici. En effet, une lecture plus positive du phénomène NIMBY suggère que la position du public sur les problèmes d'implantation peut être rationnelle et politiquement légitime. Les citoyens ont alors une bonne compréhension des problématiques ainsi que des inquiétudes légitimes pour certains risques réels planant sur la santé et le bien-être de la communauté ayant pu être ignorés par les promoteurs et politiques. Selon cette perspective, l'opposition locale sert de ce fait l'intérêt général, par exemple en identifiant des faiblesses dans les analyses d'experts concernant les propositions de sites d'implantation ou en proposant des alternatives crédibles au projet.

La récurrence des contestations locales ces dernières années peut être reliée à: (1) l'adoption par le grand public de nouvelles valeurs environnementales; (2) une augmentation des informations accessibles au public concernant les risques sanitaires et environnementaux des installations ; $(3)$ une perte de confiance dans la capacité du gouvernement et des experts à prendre des décisions informées, prudentes et équitables sur les technologies risquées; (4) la création statutaire de nouvelles opportunités pour la participation publique dans les processus administratifs et judiciaires. Le NIMBY s'est alors ramifié et de nombreuses expressions ont vu le jour autour des mouvements locaux ${ }^{5}$. Précisons que si au Nord, les

\footnotetext{
${ }^{5}$ NIABY (Not In Anyone's Backyard - Dans l'arrière-cour de personne) ; NOPE (Not On Planet Earth - Pas sur la planète Terre) ; NIMTOO (Not In My Term Of Office - Pas pendant mon mandat) ; CAVE (Citizens Against Virtually Everything - Citoyens contre virtuellement tout) ; NOTE (Not Over There Either - Pas là bas non plus) ; BANANA (Build Absolutely Nothing Anywhere Near Anyone $\mathrm{Ne}$ construisez rien nulle part près de qui que ce soit).
} 
Nimbistes sont considérés comme des opposants aux nouveaux projets ayant une vision étroite et égoïste de la situation, dans les pays du Sud toutefois, le NIMBY a une signification très différente qui est fortement liée au pouvoir communautaire et à la démocratie populaire. Les contestations locales peuventelles être l'expression d'un mouvement social enrichissant la démocratie et créant de nouvelles valeurs territoriales ? C'est ce à quoi nous allons tenter de répondre en analysant un cas d'opposition à une décharge dans un village rural de France.

\section{Etude de cas : le projet de décharge à St-Escobille, Essonne}

Le projet de décharge qui nous intéresse ici touche St-Escobille, une petite localité rurale d'Ile de France située à l'extrême sud-ouest du département de I'Essonne (91) au cœur de la Beauce, une des plus grandes régions agricoles de France. St-Escobille a la particularité d'avoir supporté les ordures de la ville de Paris pendant plus de 50 ans, depuis l'installation pendant la première guerre mondiale d'un dépôt de gadoues et d'ordures par la STIRU 6 , sans aucune forme d'autorisation. «Tous les matins, 35 wagons venus de Paris repartent après avoir basculé leurs contenus à St-Escobille. C'est un flot où se trouve inscrit absolument tout de la vie parisienne, depuis le premier mégot jusqu'à la capote anglaise », écrit l'écrivain Michel Tournier dans son ouvrage Les météores en 1975. Il faut attendre 1992 pour qu'une société s'installe et parvienne à valoriser cet ancien dépôt illégal, en le transformant en zone de production de terreau végétal tout en créant 20 emplois locaux. St-Escobille sort juste de ce combat d'un siècle contre un certain lobby parisien partisan du stockage de déchets en grande banlieue. Selon les acteurs locaux, le passé de La Gadoue ne peut être invoqué pour justifier l'implantation d'une nouvelle décharge, bien au contraire; « on a assez donné ${ }^{7}$.

Et pourtant, une habitante d'un village voisin propose en 2002 ses terres d'une superficie de 19 ha situées sur la commune de St-Escobille à une grande entreprise, alors en prospection. Sur ces terres agricoles, I'industriel souhaite installer un CSDU de classe 2 visant à enfouir 150.000 tonnes de déchets industriels banals (DIB) par an sur une période de 10 ans. Si aucune discussion ne s'est engagée entre l'entreprise et les acteurs locaux, ces derniers, en revanche se sont rassemblés pour tenter d'organiser une forme de résistance au projet que tous jugent inapproprié. Fin 2002 est créée une association de défense loi 1901 dénommée Association de Défense de St-Escobille (ADSE), dont le Président est à l'époque le Maire de StEscobille et qui compte une centaine d'adhérents. Dix ans plus tard, I'ADSE, présidée par la mairesse de Mérobert (commune voisine de St-Escobille), compte un millier de membres et s'investit dans des problématiques similaires partout en France.

Notre objectif est de décrypter l'organisation de cette résistance et les effets qu'elle génère sur le territoire à travers une analyse du jeu d'acteurs autour du projet de décharge. En premier lieu a été réalisée une synthèse bibliographique d'un côté sur les impacts d'un centre d'enfouissement sur un territoire et de I'autre sur le phénomène NIMBY. En second lieu, notre présence sur le terrain en 2007, 2009 et 2011 nous a permis d'analyser l'évolution des modes de résistance dans le temps au-travers une démarche d'observation participante. Finalement, afin de réunir des informations aussi délicates que celles traitant des perceptions ou des rapports de force entre parties prenantes, il est apparu nécessaire de réaliser une série d'entretiens avec les acteurs concernés par le projet (tableau 1).

\begin{tabular}{|l|l|}
\hline Observation participante & Entretiens semi-directifs \\
\hline $\begin{array}{l}\text { Présence sur le terrain en 2007, 2009, 2011 } \\
\begin{array}{c}\text { Participation à de nombreux évènements } \\
\text { (manifestations, réunions, débats entre }\end{array}\end{array}$ & $\begin{array}{c}\text { Entretiens auprès de } 3 \text { experts et } 5 \text { membres de l'ADSE (Les } \\
\text { citations dans le texte correspondent aux discours des } \\
\text { membres de I'ADSE) } \\
\text { Guide d'entretien élaboré à partir de la stratégie patrimoniale }\end{array}$ \\
\hline
\end{tabular}

\footnotetext{
${ }_{7}^{6}$ Société de Traitement Industriels des Résidus Urbains

${ }^{7}$ Les entreprises identifient les anciens dépôts illégaux pour s'installer car l'acceptabilité sociale du déchet est en général facilitée.
} 
Tableau 1 : Aspects méthodologiques

\section{III - Résultats : quand la résistance s'éclaire de 4 types de capitaux}

En tant qu' «acteurs faibles », c'est-à-dire des humains contemporains sous-représentés (Sébastien, 2011), non impliqués dans les négociations, les riverains souhaitent à l'origine faire entendre leur avis et lutter de manière dynamique contre la construction du centre d'enfouissement « pour éviter que la région ne devienne la poubelle de l'Ile de France ${ }^{8} \gg$. Passé le rejet instinctif face à I'appropriation d'un espace quotidien (Jobert, 1998), les logiques de mobilisation se structurent vite, car il s'agit d'inscrire les revendications dans une cause collective (Lolive, 1997). Quatre types de capitaux découlent de cette opposition locale au projet industriel : social, savant, patrimonial et politique.

\section{Une résistance créatrice de capital social}

Le capital social, selon les travaux de Robert Putnam, est défini par les richesses des réseaux qui connectent entre eux les membres d'une société et les normes de réciprocité et de confiance qui en découlent (Putnam, 2000). Sa production est le fruit de relations qui se nouent au sein d'une structure sociale et découle d'organisations sociales qui rendent possible la réalisation d'objectifs difficilement atteignables en dehors. La résistance au CSDU de St-Escobille a créé un capital social important sur le territoire au travers notamment de trois réseaux sociaux.

Le premier objectif de l'association fut de prendre contact avec une série d'experts afin d'acquérir les connaissances nécessaires à l'analyse des impacts de la décharge sur le territoire. D'abord, un avocat pour les aspects réglementaires, puis un ingénieur à propos des défaillances des géomembranes, suivi d'un médecin pour les aspects liés à la santé et un hydrogéologue sur les aspects techniques. Petit à petit, retraités et actifs, élus et agriculteurs parviennent à trouver les bons interlocuteurs, s'entourent des meilleurs experts et se noue alors un premier réseau technique autour de la lutte contre le projet de décharge.

Au niveau des impacts sur la faune et la flore, I'ADSE a souhaité contacter plusieurs associations de protection de la nature (APN), dont la Ligue pour la Protection des Oiseaux (LPO) ou Essonne Nature Environnement (ENE). Mais au premier abord, les beaucerons ont trouvé une fin de non recevoir. En effet, quel paradoxe pour les APN de soutenir le paysan beauceron, le symbole même de l'agriculture intensive, pointé du doigt comme le grand responsable de la dégradation de l'environnement régional. Les APN furent réticentes à soutenir les Beaucerons dans leur combat ...environnemental et jugeaient que, quitte à créer une décharge, il n'était pas illégitime de l'installer au cœur de la Beauce, zone déjà sinistrée pour la biodiversité. Mais l'ADSE n'a pas relâché sa pression, et a fini par prouver à ses interlocuteurs qu'il ne s'agissait pas là de riverains souhaitant conserver leur cadre de vie mais d'acteurs locaux assidûment mobilisés dans la démonstration de l'incongruité d'un tel projet en Essonne du sud. Ayant assis sa légitimité, I'ADSE a finalement reçu le soutien officiel de plusieurs APN sur ce dossier, sans toutefois aller trop loin dans les collaborations. En effet, les conflits de valeur morale à propos de la gestion de la nature freinent les actions communes. "Si des APN trop vertes adhéraient à I'ADSE, certains agriculteurs quitteraient l'association; en même temps, certains écolos sont odieux avec les Beaucerons. C'est l'occasion ici d'entamer le dialogue ». Il s'agit là néanmoins d'une victoire pour les riverains mais également d'un rapprochement entre deux mondes a priori antinomiques : l'écologie et la ruralité. Se crée alors un second réseau, dit associatif, auquel viennent rapidement s'adjoindre de nombreuses associations liées à la gestion des déchets ou à la protection de l'environnement en France mais aussi à l'étranger. On recense sur le site internet de I'ADSE 42 références à des sites associatifs contre les centres

\footnotetext{
${ }^{8}$ www.adse.fr
} 
d'enfouissement ou pour la qualité de vie, 9 sites de grandes associations de protection de la nature en France ainsi que des sites de lutte anti-décharge en Belgique, au Canada et aux Etats-Unis. L'ADSE est d'ailleurs souvent sollicitée par d'autres associations dans le même cas pour leur transmettre son savoirfaire. "On est dépassés par notre succès! On a récemment aidé une association a annuler un projet de décharge; à croire qu'on est plus efficace ailleurs que chez nous ; c'est sans doute ça l'anti-NIMBY! [rires]».

Finalement, un réseau local prend racine avec la création de l'association. St-Escobille abrite les agriculteurs d'un côté, et les rurbains de l'autre, des habitants de la commune travaillant à Paris; deux mondes sans communication jusqu'alors. Jusqu'au projet de décharge, les habitants de St-Escobille vivaient chacun chez soi, comme c'est le cas dans de nombreux villages agricoles, qui plus est lorsqu'ils sont situés dans la couronne parisienne jalonnée de zones-dortoirs. Le projet de décharge a amené à réunir ces deux populations qui ont dû collaborer dans une lutte commune. Ainsi lors des réunions hebdomadaires travaillent côte à côte élus de tous bords politiques, exploitants agricoles, écologistes et citadins, des acteurs aux visions antagonistes et qui, sans la décharge, auraient eu peu de chance de se croiser. Ce type d'association -au sens sociologique du terme comme action visant à faire exister un collectif- a pour effet la production de lien social, des individus ayant dû accepter de se parler et d'élaborer conjointement un argumentaire commun, au point qu'une association - au sens juridique de la loi 1901 - issue d'initiatives plurielles et hétérogènes, puisse les représenter en tant qu'interlocuteur auprès des pouvoirs publics. Les espaces participent alors de la construction du capital social tout comme ils I'induisent (Ghorra-Gobin, 2001); en développant des réseaux de sociabilité dans le passage à l'action collective, cela donne à voir des solidarités peu habituelles et des proximités parfois peu ordinaires. Réseaux technique, associatif et local, tel est l'effet paradoxal de la décharge à St-Escobille: elle a créé du dialogue social entre acteurs sur le territoire.

\section{Une résistance créatrice de capital savant}

Le capital savant peut être défini comme l'ensemble des connaissances acquises (scientifiques, vernaculaires ou institutionnelles) au cours du processus de résistance. Quand les profanes acquièrent un capital savant important, ils sont en mesure de questionner les études fournies lors de l'étude d'impact en critiquant de manière scientifique les données, méthodologies et aspects techniques du projet. Les initiatives citoyennes peuvent également générer des contrexpertises, situations appelées « duels scientifiques » (Busenberg 1999). C'est précisément ce qu'il s'est passé ici.

D'abord, I'ADSE met le doigt sur les incertitudes radicales associées aux décharges, comme les fuites de la géomembrane, laquelle cesserait d'être efficace comme barrière de contaminant entre 0 et 4 ans après I'installation (Rowe et al, 2003); les écoulements de lixiviats toxiques, métaux lourds dissous par traitement anaérobie (Vilomet, 2000) ; I'évacuation de biogaz, principalement composé de méthane, très problématique pour le réchauffement climatique (Binder \& Bramrud, 2001). En outre, I'association compile l'ensemble des études qui portent sur les pathologies touchant les riverains des centres d'enfouissement (Fielder et al, 2000) (leucémies, cancers de la vessie et de l'estomac chez les adultes et anomalies de naissance chez les enfants) et montre que les décharges ont un impact évident sur la qualité de vie des riverains et sur les prix des logements situés à proximité (Bouvier et al, 2000).

Ensuite, I'ADSE montre que le rapport fourni par l'industriel néglige le fait que le plateau de Beauce, et particulièrement au niveau du site projeté, est un système hydrologique karstique, qui plus est situé sur une des ramifications de tête de la vallée sèche de la Louette. Pourtant, la grande perméabilité des sols du plateau de Beauce n'est plus à démontrer (BRGM, 2004); dès 1911, le géographe Vidal de la Blache parle de la Beauce comme d'un «calcaire fissuré et perméable incapable de retenir les eaux». "On s'est cotisés pour demander une contrexpertise au BRGM, qui a découvert une zone de fracture sur le site, non évoquée par l'industriel, comme par hasard». En allant plus loin, l'association découvre que les mesures de perméabilité réalisées par l'entreprise sont presque inutilisables et recense de nombreux captages et 
puits dans un rayon de $5 \mathrm{~km}$ autour du projet de décharge. Les membres de l'ADSE ont également analysé la présence de zones classées à proximité, d'espèces protégées (faune/flore), l'insertion paysagère, l'impact visuel, les nuisances olfactives et les vents dominants, pour s'apercevoir que l'étude d'impact se contente de survoler l'ensemble de ces points qui sont, selon I'ADSE, d'intérêt général. Toutes ces lacunes scientifiques ont été dans un premier temps relevées par des profanes puis confirmées ensuite par le bureau d'étude en charge du dossier. «Maintenant, on m'appelle Docteur ès Ordures quand j'arrive en réunion».

Au fil des recherches scientifiques et des discussions avec les experts, les habitants du sud-Essonne se sont rendus compte que non seulement un CSDU classe 2 n'a pas sa place sur l'emplacement choisi à StEscobille mais aussi que construire une décharge bête et méchante au XXIème siècle est une aberration, compte tenu des évolutions technologiques. En effet, les membres de I'ADSE ont étudié les meilleures technologies disponibles des autres pays ou régions de France, et ainsi les avantages et inconvénients de la thermolyse, la méthanisation ou le tri mécano-biologique en se rendant sur plusieurs sites européens utilisant ces techniques. "Dans tri mécano-biologique, le mot biologique est une foutaise [...] bon, on voyait la thermolyse d'un bon œil au début mais on a changé d'avis après notre visite à l'usine d'Arras, qui a fermé depuis! [...] la combustion anaérobie fonctionne pour de mini-unités seulement». Parmi les différentes propositions de l'association, on trouve la biométhanisation à certaines conditions, la mise en place de petites unités de traitement à l'échelle communale, la stabilisation des déchets avant enfouissement, le tri des déchets par ballot, le développement des ressourceries et évidemment davantage d'obligations pour les industriels ainsi qu'une réduction globale de la production des déchets.

Finalement, au cours des démarches engagées, un savoir institutionnel se développe. Le collectif ainsi formé apprend beaucoup sur l'existence, le rôle et le fonctionnement des institutions gravitant autour du dossier, débrouille les compétences des divers niveaux électifs, des administrations et de leurs services techniques. Géomembrane, lixiviat, biogaz, méthanisation mais aussi enquête publique ou recours administratif n'ont plus de secret pour les riverains du projet qui travaillent depuis plus de dix ans sur le dossier. Ils ont ainsi développé et mobilisé un panel de connaissances à la fois scientifiques, politiques et procédurales concernant la gestion des déchets en France, et plus spécifiquement sur la question des centres d'enfouissement. L'ADSE participe systématiquement à toute conférence sur le thème des déchets ayant lieu en France et en profite pour interpeller les scientifiques sur le sujet. "On revient d'un colloque au Muséum avec lequel l'industriel collabore pour promouvoir la biodiversité ! Un comble; on les a interpellés mais ils nient en bloc. C'est vrai que la biodiversité associée aux décharges est des plus intéressantes... ». On assiste alors à une réduction de la distance entre les visions expertes et profanes avec I'apparition d'un public composite, pluriel, dépositaire de savoirs, de connaissances et de compétences spécifiques liées à son expérience et à ses habiletés qui le rend à même d'accomplir des « expertises profanes »(Doidy, 2003). Ces différentes connaissances constituent ce que nous appelons le capital savant initié par le mouvement de résistance.

\section{Une résistance créatrice de capital patrimonial}

Nous appelons capital patrimonial l'ensemble des attachements au territoire dans l'espace (la nature) et dans le temps (la tradition). Si la mobilisation démarre au nom d'un attachement local, elle crée davantage d'attachements au fur et à mesure que la résistance avance. Dans ce cas, le projet de décharge constitue un élément perturbateur qui fait (ré)exister le territoire local ; le projet sert d'analyseur de I'implicite territorial. En effet, parallèlement aux recherches scientifiques et aux différentes expertises, I'ADSE organise de nombreuses manifestations en tous genres afin de rassembler toujours davantage d'adhérents autour de la résistance ; afin aussi de s'encourager pour rester mobilisés. Pendant ce temps, les articles dans la presse s'accumulent ${ }^{9}$. Soirées à thèmes, concerts, ventes aux enchères, journées du patrimoine, débats ou repas dansants sont autant d'exemples qui soulignent la volonté des membres de

\footnotetext{
${ }^{9}$ On dénombre en août 2009101 articles de presse touchant au projet de CSDU à St-Escobille
} 
I'ADSE de ne pas se laisser... enterrer. De même, depuis la fondation de l'association en 2002, une dizaine de personnes de I'ADSE se réunit chaque lundi soir pour avancer sur le dossier; cela démontre à quel point le mouvement de résistance est tenace.

En plus des rapprochements sociaux, cet engagement des adhérents a conduit à des effets rebonds inattendus. L'association a par exemple découvert un site gallo-romain sur la commune. De même, une soirée a été organisée sur le thème des Gadoues de St-Escobille où de nombreux intervenants ont témoigné à propos de l'ancien dépotoir illégal. "Les rats attaquaient les chevaux et on voyait des feux spontanés partout. On ne peut pas revivre ça. » Enfin, I'ADSE a défendu des zones humides délaissées du territoire, ainsi que le busard cendré. La lutte contre le projet de décharge aura contribué indirectement à la remise en valeur du territoire de la Beauce, à la redécouverte de l'histoire et du patrimoine, à la création d'attachements territoriaux. Ces derniers permettent une montée en généralité par le partage de l'indignation suscitée par le projet. Aussi étonnant que cela puisse paraître, les membres de l'ADSE ont d'abord ancré leur attachement au territoire, puis ont réussi à le transmettre à d'autres acteurs, en présentant «leur Beauce » différemment des clichés habituels. "Elle est quand même belle la plaine ; on doit plus communiquer sur le territoire car notre Beauce, c'est une mal-aimée, à tort . » L'introduction d'un élément extérieur (le projet de décharge) peut servir de révélateur des tensions locales et susciter une réflexion sur l'avenir d'un territoire, lequel symbolise la volonté de vivre ensemble. La résistance au projet a ainsi redonné un sentiment d'appartenance des acteurs à cet espace au travers de la constitution d'un capital patrimonial.

\section{Une résistance créatrice de capital politique}

Nous définissons le capital politique comme la capacité d'un acteur à se positionner sur la scène politique, basée sur sa crédibilité (apports de connaissances valides), sa pertinence (réponse à des besoins dans les sphères décisionnelles) et sa légitimité (représentativité de l'acteur sur le territoire). D'abord et selon Bourdieu (1980), la conquête d'un capital social demande la constitution et l'entretien d'un « réseau durable de relations » qui peut se transformer en capital politique. Nous soutenons que le capital social est nécessaire mais non suffisant pour se transformer en capital politique ; ce sont les capitaux savant et patrimonial qui permettent au capital social de se muer en capital politique ${ }^{10}$. C'est ce qui $\mathrm{s}^{\prime}$ est produit à St-Escobille. Toujours selon Bourdieu, l'accès au pouvoir au sein du champ politique reste tout d'abord subordonné à l'existence d'un certain capital politique qui confère une autorité spécifique ; dans le cas qui nous occupe, cette autorité a pu être légitimée grâce à la constitution des trois premiers types de capitaux. En effet, I'ADSE mobilise ces différents capitaux d'abord pour s'opposer au projet puis pour proposer certaine alternatives ; ces propositions s'effectuent dans un premier temps au niveau local, puis régional et aujourd'hui sur le plan national. Fait marquant dans cette évolution, I'ADSE, acronyme initial en 2002 d'Association de Défense de St-Escobille devient en mai 2011 I'Association de Défense de la Santé et de l'Environnement, preuve d'un souhait des membres de monter en généralités.

Le premier acte de positionnement politique de I'ADSE, et donc de montée en généralités, survient en 2005, lorsque l'association rédige un «Moratoire sur les déchets d'enfouissement et de stockage de déchets ultimes », qu'elle diffuse à l'ensemble des acteurs du territoire, aux institutionnels, aux élus et aux associations. Ce moratoire est basé sur 4 points: (1) redéfinir la notion de déchet ultime en retirant les ordures ménagères ainsi que certains déchets du BTP afin de réduire la masse de déchets réellement non valorisables; (2) réévaluer les gisements de déchets ultimes dont une part importante peut être valorisée; (3) rechercher des procédés technologiques innovants en matière de déchets ultimes; (4) abandonner tout projet industriel d'enfouissement pouvant mettre en péril les nappes souterraines. Le moratoire a reçu un écho favorable dans la région, ce qui a encouragé les membres à persévérer. L'enquête publique a d'ailleurs rendu un avis défavorable au projet de modification du plan d'occupation des sols (autoriser un

\footnotetext{
${ }^{10} \mathrm{Ce}$ capital politique se rapproche de ce que certains nomment le capital militant, voir Matonti F et Poupeau F, « Le capital militant. Essai de définition ", Actes de la recherche en sciences sociales, 2004/5 no 155, p. 4-11.
} 
centre d'enfouissement sur des zones agricoles). Néanmoins, le préfet a demandé que le projet soit classé d'intérêt général (PIG), contre l'avis de la Région Ile de France, compétente en matière de planification d'élimination de déchets. L'ADSE a effectué des recours contre cet arrêté et est soutenue officiellement par 27 élus toutes échelles confondues, 132 communes sur plusieurs départements, 11 groupements agricoles et 43 associations. Pour les militants, il s'agit d'un déni de démocratie où l'industriel et le préfet veulent à eux seuls imposer cette décharge coûte que coûte contre l'avis de tous. «En se battant contre le projet, on a découvert toutes les fourberies des procédures juridiques et administratives; on dirait que le système fait tout pour décourager le citoyen à se faire entendre. On est indignés ».

Parmi les faiblesses du dossier de l'industriel, les acteurs locaux ciblent nombre de contradictions avec le PREDMA $^{11}$, le Plan de Gestion des Déchets du Bâtiment (PGDB), l'Agenda 21 départemental, le Plan de Déplacement Urbain (PDU) et le SAGE ${ }^{12}$ de la nappe de Beauce. Sur ce dernier point, I'ADSE a participé activement à l'enquête publique liée à la révision du SAGE de Beauce, étant donné qu'il s'agit de I'un des rares documents opposables où la concertation est réellement intégrée. "Pendant le dossier SAGE, je dormais $2 h$ par nuit. On souhaite y intégrer une norme sur l'entrepôt de déchets mais ce n'est pas évident de maîtriser toutes les procédures à suivre».

Les membres de l'ADSE souhaitent une politique qui place le citoyen au cœur du système selon un schéma équilibré et de proximité, de nature à créer un effet de conscientisation des enjeux locaux en donnant à chacun des outils pour participer concrètement à un développement durable. La présidente de I'ADSE est d'ailleurs allée défendre ce propos à la Commission des Pétitions du Parlement Européen en mars 2011 ; le dossier enregistré doit aboutir à une enquête pour voir si la France suit bien les prérogatives européennes en matière de gestion des déchets. L'ADSE a également effectué deux recours au Conseil d'Etat pour faire annuler un décret sur la gestion des déchets suite au Grenelle et l'autre sur le durcissement des statuts pour les APN. Des citoyens qui s'opposent et qui proposent ; voici l'exemple d'un front de refus qui sort du jardin privé pour mener un travail politique de fond et ce, pour une cause commune. Un capital politique important initié par une contestation locale. "Nous ne sommes pas des Nimbistes; on ne peut pas s'opposer à la décharge sans faire de propositions. Cette lutte nous a ouvert les yeux sur tous les problèmes environnementaux auxquels font face les acteurs locaux, pas seulement les décharges. Maintenant qu'on les connaît, on est obligés d'agir ».

\section{IV - Discussion}

\section{Qui est légitime à définir l'intérêt général ?}

Un premier point à soulever est celui de l'intérêt général. En effet, l'arrêté préfectoral visant à faire passer le projet de décharge en PIG, contre l'avis des associations et élus de tous échelons, révèle un dysfonctionnement au niveau de la définition de l'intérêt général et une perte de légitimité des acteurs le définissant traditionnellement. "Le préfet veut maintenant pouvoir modifier le POS sans consultation des habitants! Une honte. Vu la situation de prise en otages des locaux, notre stratégie est de saisir toutes les occasions pour s'opposer au caractère d'intérêt général imposé par le préfet et de prouver qu'il s'agit au contraire d'intérêt privés! On reste dans la légalité mais s'ils nous poussent à bout, on serait partant pour aller plus loin». Ainsi, l'opposition entre intérêt général et intérêts particuliers qui apparaissait évidente hier semble aujourd'hui de moins en moins pertinente pour décrire les tensions dans l'aménagement du territoire ; on assiste à des déplacements des frontières entre intérêts légitimes, où la légitimité technicoéconomique est de plus en plus contestée (Jobert, 1998). Les intérêts particuliers tendent à s'effacer

11 Plan Régional d'Elimination des Déchets Ménagers et Assimilés

12 Schéma d'Aménagement et de Gestion des Eaux 
derrière une (relative) égalisation des légitimités (légitimités scientifique et technique, de représentation et de proximité).

Comme à St-Escobille, la montée en généralités est un mécanisme de construction de l'intérêt général par les associations. En effet, les contestations de proximité soulignent la difficulté contemporaine à penser les autorités publiques comme les seuls dépositaires de l'intérêt général dans un monde que l'on redécouvre instable et incertain, en éprouvant les conséquences (in)attendues, (in)directes et persistantes de la modernisation de l'espace et de l'action publique (Latour, 2003). Comme le dit Jobert, 1998, il est important de souligner l'évidence de l'intérêt territorial et de la légitimité nouvelle des acteurs locaux à le défendre, les intérêts territoriaux (c'est-à-dire en présence sur un même espace) étant premiers dans la définition de l'intérêt général.

Les conflits d'usage constituent des marqueurs des évolutions sociales, techniques et économiques et constituent autant de tests sur la qualité et la recevabilité des décisions d'aménagement. Le travail des militants devra consister en la mise en forme d'un bien commun universalisable susceptible de concurrencer l'intérêt général défini par les autorités traditionnellement habilitées à le déterminer. Les inquiétudes locales ne sont pas en contradiction avec le bien commun, bien au contraire. C'est au travers de l'expression des inquiétudes locales que l'on parvient à comprendre le bien commun (McAvoy,1998).

Alors que l'argument NIMBY ramène la revendication à une défense d'intérêts privés, les militants ont su la transformer en une redéfinition de l'intérêt général. On rejoint une idée chère à Amartya Sen, 2003: il est possible de corriger les défauts de la démocratie et les imperfections de la prise de décision grâce aux réactions des populations qui intègrent ces éléments dans leurs bases d'information.

\section{Vers un mouvement Ni Ici Ni Ailleurs}

Les formes actuelles que prennent les mobilisations constituent d'improbables dynamiques collectives et semblent relever d'un engagement plus politique qu'il n'y parait. Le cas de l'opposition au projet de décharge à St-Escobille s'inscrit dans cette lignée, car la résistance a été éclairée par quatre capitaux : social, savant, patrimonial et politique. Tenter de caractériser ces quatre types de capitaux a le mérite de relancer le débat sur les fondements sociaux du lien politique et les rapports entre société civile et démocratie. Alexis de Tocqueville voyait dans l'association volontaire de citoyens la clé de la vitalité démocratique, qui élargit I'horizon des participants, stimule la prise de parole et le débat, apprend à agir en commun sur un pied d'égalité. Dans la même ligne, Almond et Verba en 1963 ont montré que la culture civique prend sa source dans les relations interpersonnelles, notamment dans les groupes secondaires comme les associations. La participation à ces dernières, sur la base de relations horizontales et égalitaires, augmenterait la confiance en autrui, le sentiment de compétence politique, le désir de participer à la vie de la Cité et l'adhésion aux valeurs démocratiques. On est loin du phénomène NIMBY.

A St-Escobille, des agriculteurs, habitants ou élus présentent une vision globale de la situation du déchet en France et en Europe ; on passe donc d'un phénomène Pas Dans Mon Jardin à un mouvement Ni Ici, Ni Ailleurs ou $\mathrm{NIABY}^{13}$. "Une association créée pour lutter contre un projet se dissout après. A I'ADSE, notre objectif est de durer dans le temps, même si le projet de décharge est annulé. Vu la connaissance acquise au niveau juridique et technique, et les problèmes similaires partout en France, nous considérons que nous avons une mission. Notre changement de nom est plus qu'un symbole; nous voulons avancer sur une meilleure prise en compte des acteurs locaux dans la gestion de l'environnement ». A ce propos, la littérature souligne que plus une association est permanente dans le temps, plus elle défend des intérêts diversifiés et spatialement étendus, et plus elle présentera un visage désintéressé, celui de intérêt général et de l'altruisme (Trom, 1999).

Les promoteurs négligent souvent la phase de consultation des habitants; pourtant, il n'est pas raisonnable de croire qu'on peut mener un projet de centre d'enfouissement dans un certain anonymat en milieu rural. Si les critères techniques sont fondamentaux, le fait incontournable est qu'un tel projet

\footnotetext{
${ }^{13}$ Not in Anybody's Backyard (Traduction de : dans aucun jardin)
} 
d'implantation doit être accepté par les élus concernés et par la population au-travers d'une réelle coécriture d'un diagnostic partagé et non d'une simple consultation. Car in situ, la consultation est parfois comprise par les riverains comme un « piège délibératif » (Blondiaux, 2001), une version symbolique de la concertation où les acteurs forts refusent la redistribution d'une partie du pouvoir de décision au profit d'acteurs faibles ou émergents (Sébastien, 2011). Les citoyens militants ont conscience que s'ils participent aux délibérations organisées par les acteurs forts, la probabilité qu'ils puissent s'y faire entendre serait faible, car les techniques de participation peuvent aussi servir de techniques de domination. Ainsi I'ADSE a-t-elle refusé de faire partie de certaines réunions, car pour les militants il s'agissait déjà d'un premier pas vers l'acceptation de la décharge sur le territoire. Plus que des consultations, les acteurs locaux attendent des apprentissages collectifs croisés. Si processus de consultation il doit y avoir, celui-ci doit tenir compte des informations techniques mais aussi des valeurs, du patrimoine, des perceptions des risques et des incertitudes (Burningham, 2000). Les experts échouent en effet à reconnaître les valeurs et incertitudes qui sont associés aux choix techniques alors que les citoyens peuvent être considérés comme des experts en matière de valeurs (McAvoy,1998). L'idée est alors de générer de nouveaux rapports entre l'expertise, l'exercice de la démocratie et la décision publique, autrement dit d'instaurer une gouvernance de confiance mutuelle.

\section{Conclusion}

L'installation d'un centre d'enfouissement ne peut être abordée que politiquement car elle concerne tout le système socio-économique dans lequel elle s'ancre. On y retrouve la distance qui sépare l'espace urbain et l'espace rural, l'activité industrielle et l'activité agricole, le temps court et le temps long, les savoirs profanes et experts, les représentations entre acteurs. Parce que les directives européennes sont rarement ou mal appliquées, parce que la gestion des déchets représente un domaine où les incertitudes scientifiques sont parmi les plus fortes, parce que la légitimité du décideur est affaiblie, les projets de décharge suscitent toujours une résistance massive et forte de la part des acteurs locaux.

A St-Escobille, entrer en conflit ne signifie pas seulement s'opposer ; c'est un vecteur d'intégration du citoyen à l'action administrative et au processus de décision publique locale. Les mouvements de riverains se donnent alors à voir sous la forme d'improbables dynamiques de socialisation politique, théâtre d'une activité de reformulation des problèmes, des acteurs et des enjeux. La lutte contestataire devient alors un mode potentiel de politisation des problèmes publics et ces collectifs hybrides reconfigurent les qualités politiques des espaces. Les conflits d'aménagement construisent ainsi des scènes locales qui, bien que réduites et circonscrites, posent des questions politiques. C'est à l'occasion de projets ponctuels que les citoyens en viennent à réinvestir le champ politique et à reposer des questions sur les choix d'organisation de la vie collective dans un territoire. Nous avons affaire ici à un travail politique au sens fort, de représentation et de confrontation sur projets, que nous nommons « résistance éclairée 》 (éclairée à la fois par le social, par le savoir, par le patrimoine et par le politique).

En effet, quatre types d'effets découlent de cette mobilisation citoyenne, a priori stigmatisée sous la bannière NIMBY. D'abord, cette lutte qui n'est pas terminée a conduit à la mise en place de solides réseaux techniques, associatifs et locaux (capital social). Ensuite, les membres de l'association ont développé des savoirs et compétences scientifiques, politiques et procédurales sur la question de la mise en place d'un centre d'enfouissement et ont su les mobiliser pour proposer des expertises profanes (capital savant). La mobilisation a également engendré de nouveaux attachements territoriaux, au travers d'une remise en valeur du territoire de Beauce, d'une redécouverte de l'histoire et du patrimoine local (capital patrimonial). Finalement, l'ensemble de ces capitaux mis ensemble induisent un capital politique, où le front de refus se transforme en force de proposition, les militants proposant des alternatives crédibles au projet industriel. Nous rejoignons Hunter \& Leyden, 1995, qui montrent que les citoyens inquiets par les aspects liés à la santé, à l'environnement ou simplement par le manque de confiance envers les décideurs ont des réclamations légitimes et ne sont pas concernés par le phénomène NIMBY. 
Le développement des sciences et techniques amène des incertitudes dites radicales notamment dans le domaine de l'environnement, incertitudes qui ne sont plus gérables par les institutions politiques dont nous disposons. Ces incertitudes scientifiques amènent donc à des controverses techniques mais aussi sociales car nombreux sont les choix techniques qui engagent le collectif, d'où une reconfiguration du paysage social. Pour reprendre la célèbre expression de Marcel Mauss, les nouveaux risques apparaissent comme des faits sociaux totaux, nœuds où s'enchevêtrent pratiques et débats relevant de tous les registres de la vie en société. Nous montrons ici que face à ces aux incertitudes environnementales, les mouvements de riverains montent en généralités, ce qui donne lieu à une mise en concurrence d'intérêts généraux ou d'utilités publiques. Les conflits environnementaux peuvent être l'expression de revendications légitimes et d'oppositions démocratiques à des projets qui ne font pas I'unanimité ; afin de saisir les enjeux associés aux conflits environnementaux, il s'agit de mieux formuler les processus de politisation en train de s'accomplir dans les contestations locales; de mieux questionner les modes d'accès à la construction délibérative de biens communs et les formes de participation à la vie publique.

\section{Bibliographie}

Almond G., Verba S. (1963) The Civic Culture. Political Attitudes and Democracy in Five Nations, Princeton, Princeton University Press.

Binder M., Bramrud T. (2001) Environmental impacts of landfill bioreactorcells in comparison to former landfill techniques, Water Air and Soil Pollution, $n^{\circ} 29,289-303$.

Blondiaux L. (2001) Démocratie locale et participation citoyenne : la promesse et le piège, Mouvements, n¹8, 44-51.

Bourdieu P (1980), Le Sens pratique, Paris, Les Éditions de Minuit, coll. Le sens commun, 475 p.

Bouvier R.A., Halstead J.M., Conway K.S., Manalo A.B. (2000) The effect of landfills on rural residential property values : some empirical evidence, The Journal of Regional Analysis and Policy, $n^{\circ} 30$ (2), 2337.

BRGM (2004) La nappe des calcaires de Beauce, Service géologique Régional Ile de France et DIREN de bassin Loire-Bretagne.

Brion (1991) Essential industry and the NIMBY phenomenon. Quorum Books, New York.

Burningham K (2000) Using the language of NIMBY : a topic for research, not an activity for researchers. Local Environment, vol 5, $\mathrm{n}^{\circ} 1,55-67$.

Busenberg G (1999) Collaborative and adversarial analysis in environmental policy. Policy sciences vol 32 n¹. 1-11.

Dear M (1992) Understanding and overcoming the NIMBY syndrome. Journal of the American Planning Association, $\mathrm{n}^{\circ} 58,141-149$.

Doidy E. (2003) Faire entendre la voix des usagers dans les concertations environnementales, Sociologies pratiques, $\mathrm{n}^{\circ} 7,49-64$.

Dziedzcki JM. (2003) Au-delà du Nimby : le conflit d'aménagement, expression de multiples revendications, in P. Melé, C. Larue, M. Rosemberg, Conflits et territoires, Presses Universitaires François Rabelais, MSH Villes et territoires, Tours.

Fielder H.M.P., Poon-king C.M., Palmer S.R., Moss N., Coleman G. (2000) Assessment of impact on health of residents living near the Nanty-Gwyddon landfill site: restrospective analysis, Br. Medicine Journal, n०320, 19-22.

Freudenberg W \& Pastor S (1992) NIMBYs and LULUs, stalking the syndromes, Journal of Social issues, 48 $n^{\circ} 4,39-61$.

Ghorra-Gobin C (2001) Les espaces publics, capital social. Géocarrefour. Vol 76 n¹, 5-11. 
Hunter S \& Leyden KM (1995) Beyond NIMBY: explaining opposition to hazardous waste facilities. Policy studies journal vol 23 n4, 601-619.

Jobert A (1998) L'aménagement en politique ou ce que le syndrome NIMBY nous dit de l'intérêt général. Politix n42, 67-92.

Kraft ME \& Clary BB (1991) Citizen Participation and the Nimby Syndrome: Public Response to Radioactive Waste Disposal. The Western Political Quarterly, Vol. 44, No. 2, 299-328, University of Utah, Western Political Science Association.

Latour B. (2003) Un monde pluriel mais commun, entretien avec F. Ewald, Editions de I'Aube, coll. Interventions.

Lolive J (1997) La montée en généralités pour sortir du NIMBY. La mobilisation associative contre le TGV Méditerranée. Politix vol10, n³9, 109-130.

Maillebouis C. (2003) Nimby ou la colère des lieux. Le cas des parcs éoliens, Nature, Sciences et Sociétés, vol.11, 190-194.

McAvoy GE (1998) Partisan probing and democratic decision making: rethinking the NIMBY syndrome. Policy studies journal. Vol $26 \mathrm{n}^{\circ} 2,274-292$.

Ollagnon H. (1989) Une approche patrimoniale de la qualité des milieux naturels, In Du rural à l'environnement, la question de la nature aujourd'hui, Ed. L'Harmattan, Paris.

Piller C (1991) The fail-safe society: community defiance and the end of American technological optimism. New-York, NY: Basic Books.

Putnam RD. (2000) Bowling Alone : the Collapse and Revival of American Community, New York, Simon and Shuster.

Rowe K., Sangam H., Lake C. (2003) Evaluation of an HDPE geomembrane after 14 years as a leachate lagoon liner, Canadian geothechnical journal, National Research Council of Canada, Ottawa, vol 40, n०3, 536-550.

Sébastien L (2009) Pour un diagnostic socio-environnemental sur un territoire : I'Acteur en 4 Dimensions (A4D). In Développement durable, Entreprises et Territoires, Vers un renouveau des pratiques et des outils. Editions I'Harmattan, Paris.

Sébastien L (2011) Quand les acteurs faibles et absents s'immiscent dans la négociation environnementale, Territoire en mouvement, $n^{\circ} 11,66-81$.

Trom D (1999) De la réfutation de I'effet NIMBY considérer comme un pratique militante. Notes pour une approche pragmatique de l'activité revendicative. Revue française de science politique. 49eme année. n'1, 31-50.

Vilomet J.D. (2000) Traçage des pollutions lixiviats de CET sur les eaux souterraines, Thèse soutenue le 6 décembre 2000 à I'Université d'Aix-Marseille III.

Wolsink M (2000) Wind power and the NIMBY-myth : institutional capacity and the limited significance of public support. Renewable energy, vol. $21, \mathrm{n}^{\circ} 1,49-64$. 